\title{
ESTABLISHING BIOMECHANICAL MODEL OF HUMAN BODY FOR RESTORING STANDING FUNCTIONS
}

\author{
Cheng-Liang Liu' ${ }^{1}$, Chung-Huang Yu² ${ }^{2}$ Shih-Ching Chen $^{3}$, Wen-Shan Wu ${ }^{1}$ \\ ${ }^{1}$ Department of Mechanical Engineering, National Taiwan University, Taipei \\ ${ }^{2}$ Institute of Rehabilitation Science and Technology, National Yang-Ming University, Taipei \\ ${ }^{3}$ Department of Physical Medicine and Rehabilitation, \\ Taipei Medical University and Hospital, Taipei, Taiwan
}

\begin{abstract}
This work presents a five-segment biomechanical model of the human body for paraplegics of spinal cord injury (SCI) with thoracic nerve injury. When the functional electrical stimulation (FES) system is used to restore sit-to-stand function, the biomechanical model can be used to analyze the position, force, and moment of the human body at every joint through inverse dynamics. A series of data taking from SCI patient under FES of restoring sit-to-stand function are implemented on the model. The results help realize the role of each joint and muscle in the sit-to-stand process so as to improve the rehabilitation in the future plan.
\end{abstract}

Biomed Eng Appl Basis Comm, 2004 (February); 16: 15-21.

Keywords: five-segment biomechanical model of human body, inverse kinematics, restoring standing function

\section{INTRODUCTION}

The treatment used electrical stimulation to actuate the muscle to yield functional movement in a process called functional electrical stimulation (FES). FES was performed on patients of spinal core injury (SCI) who could not stand up from the sitting position [1-2]. Some other research work attempted to use FES to restore hand functions including grasping, pinching and others [3]. However, standing from a wheel chair was a viable option for some patients. Some kinematics and dynamics analyses had described the movements of elderly or rheumatoid arthritic patients

Received: Nov 10, 2003; Accepted: Dec 25, 2003

Correspondence: Cheng-Liang Liu, Professor

Department of Mechanical Engineering, National Taiwan University, No.1, Section 4, Roosevelt Rd. Taipei 106, Taiwan

E-mail: clliu@ntu.edu.tw
[4-6]. An early research applied FES to the lower limb paralyzed that stimulated the hip muscles and quadriceps and made a $\mathrm{T} 3$ patient stand up from a sitting position [7]. In Kralj and Bajd's book [8], Wilemon and Reswick implanted penetration electrodes near the femur and hip nerves. The stimulation of the muscles extended the knee joints and hip joints. A pair of ankle ortheses were then used to stabilize the ankle joints. Kralj and Bajd had also done much work on FES research and concluded that the lower limb paralyzed could stand up only by using surface FES technique [9].

The research on standing and sitting by patients with SCI, presented by Kralj and Bajd, involved a series of electrical pulses that stimulated quadriceps, gluteus maximus, gluteus medius, soleus and gastrocnemius [8]. All these stimulation seemed to need a controller that an appropriate model was thus essential to analyze the movement of each segment 
feedback from transducers attached on the human body [10-11].

The strength of the muscle also importantly affected the sitting-to-standing process of a patient. Khang and Zajac developed a formula to describe the relationships among the strength of FES, muscle activation dynamics, musculo-tendon dynamics and body-segmental dynamics [12]. Minimal energy consumption was the goal, to reduce muscle fatigue. Graupe et al. tried to extract EMG signals from the shoulder and then controlled the movement of the lower limbs [13].

The use of auxiliary tools and other parts of the body can help standing to some extent. Veltink and Donaldson considered that a patient's autonomic nervous system would be in concert with his or her FES system [14]. Accordingly, a simpler FES controller could be developed. Donaldson and Yu designed an auxiliary system controlled by handle reactions of a stimulated leg muscle [15]. The system measured the forces and moments of the arm in concert with the lower limb underwent FES that helped the SCI T6 patient stand up and sit down.

The development of efficient strategies for standing and sitting, to design an FES controller is also an important area of research. Riener and Fuhr designed a patient-driven motion reinforcement controller [16]. They measured the position and speed of upper limb joints to control the FES of lower limbs to help SCI patients stand up and sit down. Kralj et al. measured reactions from the foot plate and the postural angles of the standing and sitting movements of normal people [17]. They classified standing and sitting movements into four stages to which different control strategies could be applied. Schenkman et al. also concluded the existence of four similar steps of standing by analyzing changes in momentum and the stability of normal people [18]. Doorenhosch et al. compared two standing strategies, natural sit-to-stand transfer and full flexion of the trunk [19]. By comparing the moment exerted by the muscles around hip joints, these researchers found that full flexion of the trunk needed a greater moment than the other strategy.

The above research mentioned about muscle forces emerged that a biomechanical model is needed to help analyze and simulate the kinematics and dynamics of joint moment, muscle strength, and movement strategy.

This work develops a model of the human body that analyzes dynamics concerning sitting-to-standing. The model 掇 inputs are signals from transducers. The outputs are full biomechanical data of human body segments and joints, such as forces, moments, displacements, velocities and accelerations. The input data was obtained from the subject of an SCI patient with only trauma beneath $\mathrm{T} 6$, the sixth thoracic nerve injury [15]. His upper limbs and muscles were still functional. An FES of lower limb muscles and functional upper limbs was performed to help the patient from sit-to-stand motion. The whole system involves two parts, the patient's upper limbs and an FES of the lower limb muscles. The electrical goniometers used to measure angles were attached to the lower limbs to collect kinematic data. One transducer transferred forces and moments from handles pulled by hands. The FES controller thus output appropriate electrical pulses to stimulate the lower limb muscles and support standing movement. The feedback signals of the goniometers and transducers were continually collected and checked by the controller. Stimulation commands were then given by the controller to FES to ensure that the standing movement follows the strategy planned.

The technique used to calculate and solve the human model is inverse kinematics. Inverse kinematics techniques have been used on a learning model that took position of a human arm posture and then feed back the error to the solver and update the joint angle [20]. Some real-time inverse kinematics techniques also used on human arm or leg that might analyze the position, orientation, and the movement constraint of the limb [21-22]. In this work, an off-line inverse kinematics technique incorporated with dynamics are used to analyze human body together with upper and lower limbs so as to perceive the full dynamics and cooperative movement behaviors of the SCI patient.

\section{CONSTRUCTING A BIOMECHANICAL MODEL}

In this work, the left and right sides of an SCI patient are assumed to be symmetrical. Thus, the modeling of the human body is a two-dimensional problem. Every joint is treated as a pin joint [23]. The head, neck and trunk of the subject are presumed in one segment. The planar kinematic diagram is shown in Fig. 1. The five segments that represent a human body are marked $\vec{l}_{2}$ to $\vec{l}_{\text {. }}$. The number " 1 " represents the fixed parts such as the ground and handle support and, $J_{1}$ is assigned as the origin. Vector $\bar{l}_{n}$ represents the $n$th segment that points from distal end to proximal end. The symbols $J_{1}$ to $J_{6}$ are joints between segments and fixed parts. The joint $J_{6}$ representrs the ankle joint and the foot is fixed on the ground. Likewise, $J_{1}$ is the wrist joint and the palm is fixed on the handle.

The degree of freedom of the model is 3 . The whole linkage can be solved with three known values. In this work, three angular positions were continually measured at the hip, knee and ankle joints during the 
sit-to-stand process. The angular velocity and acceleration were then obtained by differentiating the angular position curves.

The angle $\theta_{n}$ is the angle between the $n$th segment and the horizontal line in the $\mathrm{x}$ direction is as shown in Fig. 1. Three angles $\theta_{4}, \theta_{5}$ and $\theta_{6}$ can be measured by electrical goniometers. Vector $\bar{J}_{n}$ is defined from joint $J_{1}$ to joint $J_{n}$, thus $\vec{J}_{1}=(0,0)$ and $\overrightarrow{J_{6}}=(-b,-a)$. The positions of $\vec{J}_{5}, J_{4}$ and $J_{3}$ can be calculated from $\vec{J}_{6}, \theta_{4}, \theta_{5}$ and $\theta_{6}$. The position of the last joint $J_{2}$ could be determined by the two segments $\vec{l}_{2}$ and $\vec{l}_{3}$, and vector $\vec{J}_{3}$. Thus, all joint positions are solved kinematically.

\section{CASE STUDY}

Donaldson and Yu conducted a case study [15]. The subject was a male SCI of T6. The raw data were angular displacements measured at the ankle, knee and hip joints. Data on forces and moments were taken from the handle transducer. The sit-to-stand process was performed and the solid lines in Figs. 2 to 7

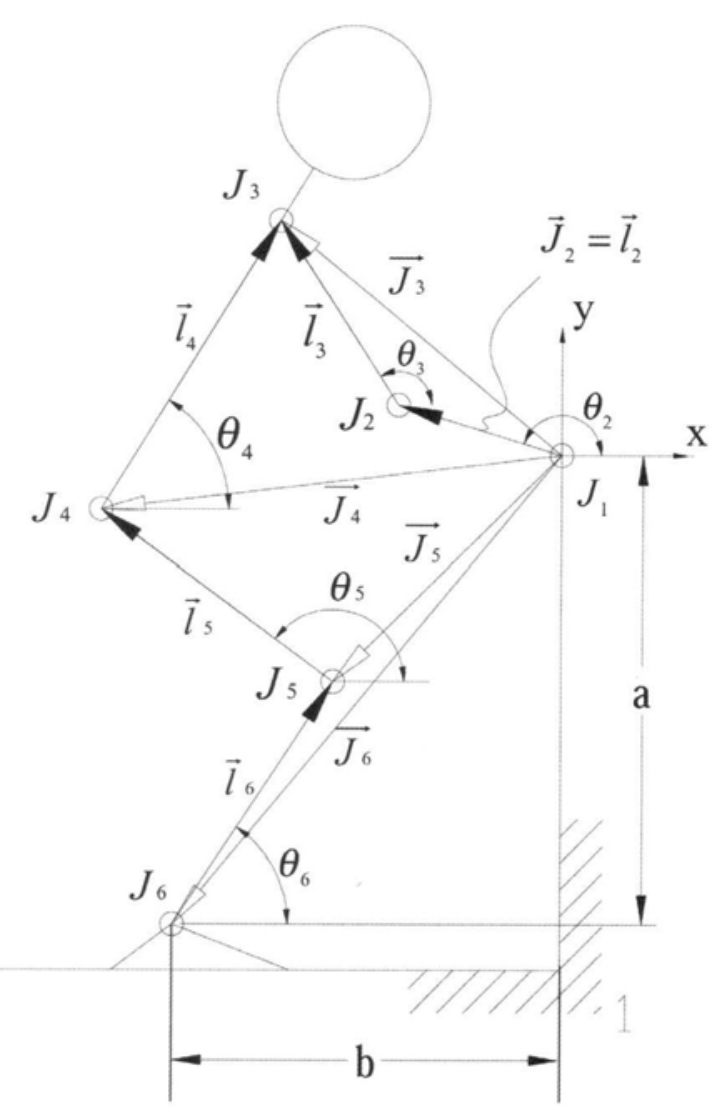

Fig. 1. Biomechanical model of human body

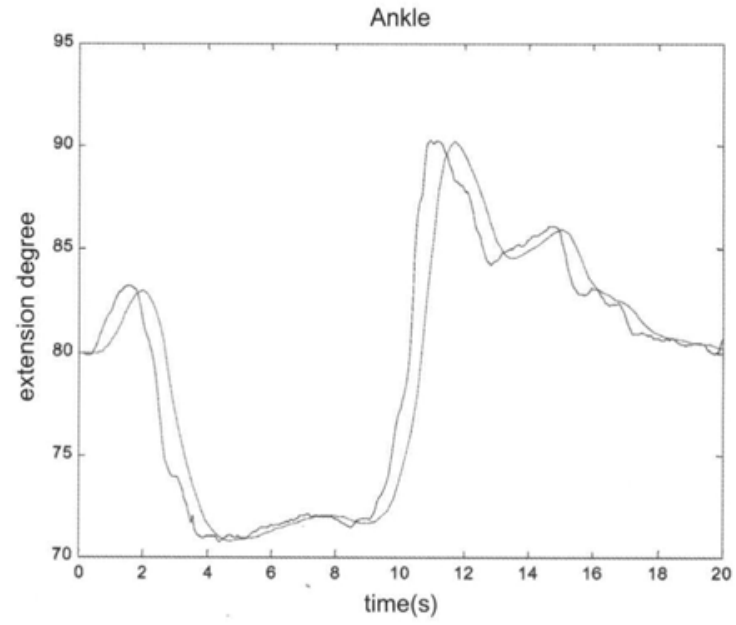

Fig. 2. Angular displacement of ankle joint

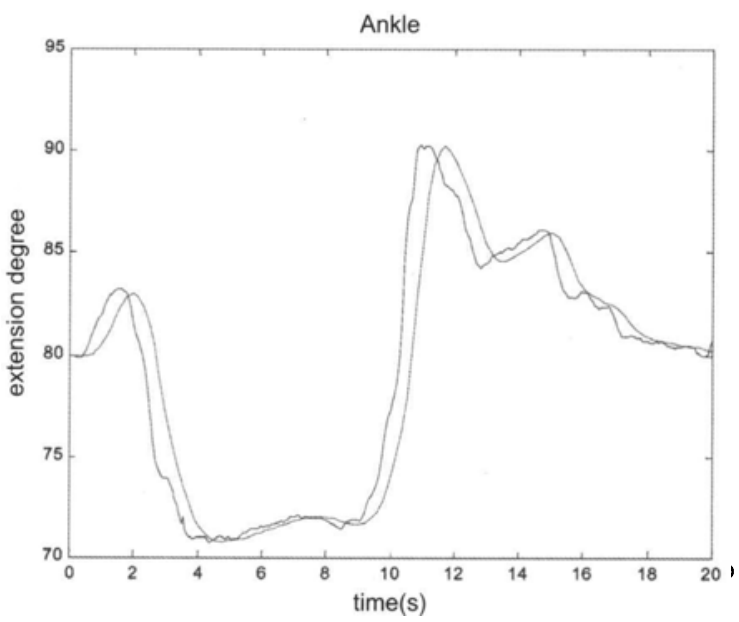

Fig. 3. Angular displacement of knee joint

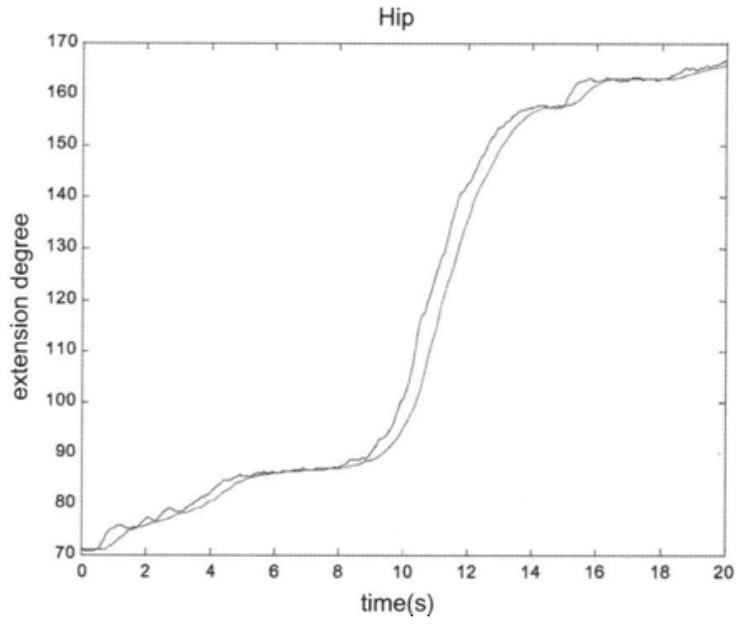

Fig. 4. Angular displacement of hip joint 


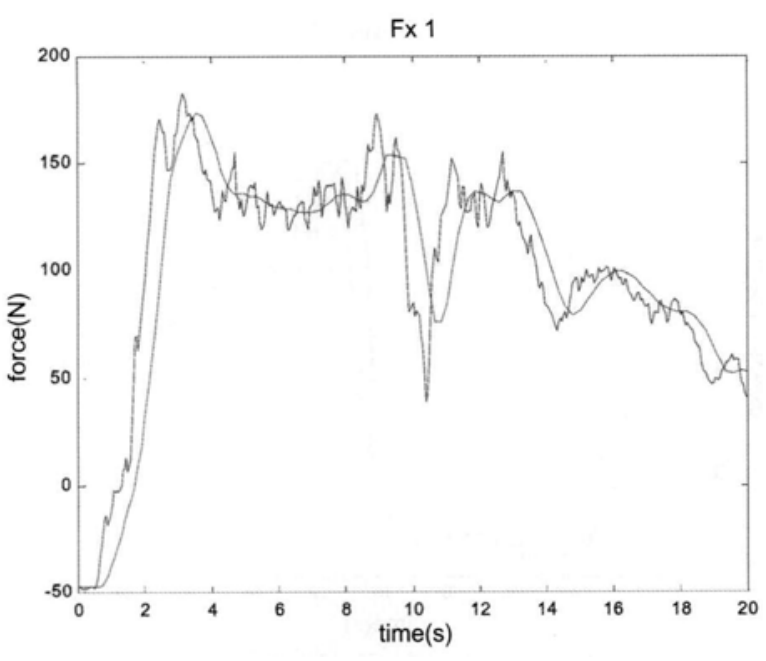

Fig. 5. Horizontal reaction on handle

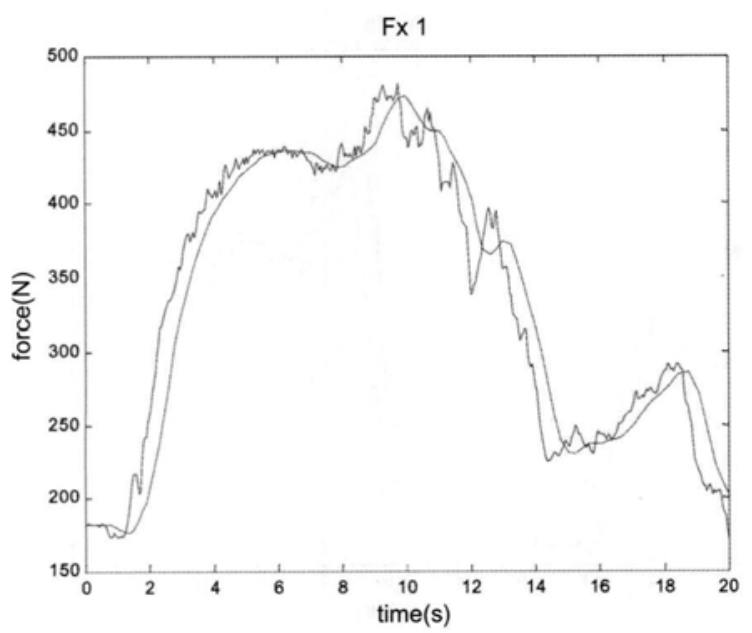

Fig. 6. Vertical reaction on handle

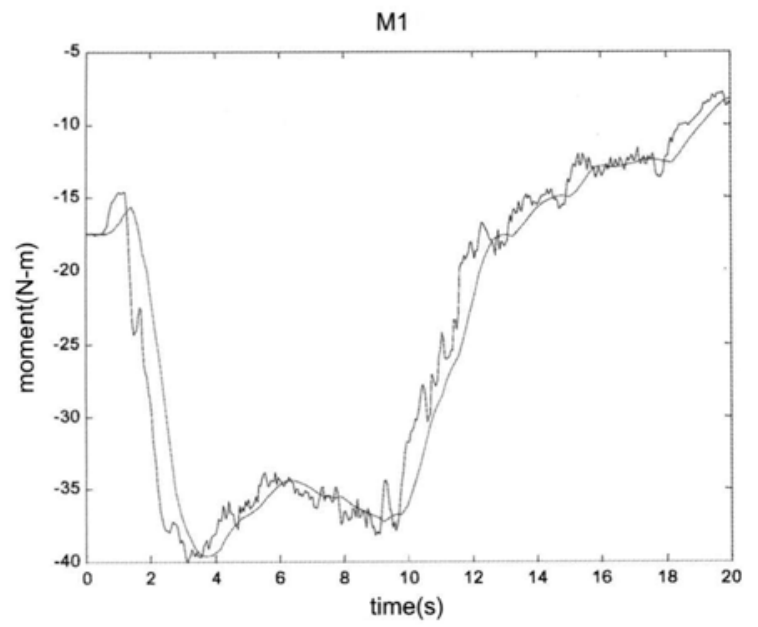

Fig. 7. Moment on handle represent the aforementioned raw data. The raw data curves with zigzag ripples may be caused by noise or shivering of the subject. These curves were then processed by a Butterworth low pass filter to eliminate the zigzag ripples. The ripple-free curves thus obtained were drawn as dashed lines in the same figures. The dashed lines, a smoothed angular displacement verses time curve, retain the trends of the raw data curves. Angular velocity curves can thus be obtained by differentiating the smoothed angular displacement curve stated above. Angular acceleration curves are then obtained by differentiating angular velocity curves. Mathematica is used as a computational tool in this work.

Figures 8 to 13 plot the dynamic results of each joint. In these figures, solid lines, dotted lines and dashed lines represent moments, horizontal forces and vertical forces, respectively. A positive moment will extend the joint and a negative value makes flexion. The vertical force that bears the weight of the subject and that the horizontal force is the balance force so as to avoid the swing of the body back and forth. The vertical force is greatest at the ankle joint that supports the whole body weight. The moments are largest at the hip and knee joints, because muscles at the hip and the thigh contributed most of the moment required to stand.

One of the figures is chosen as an example to explain the dynamics of a segment as follow. The horizontal force applied to the shoulder joint, depicted by the dotted line Fx3 in Fig. 10, changed from negative to positive, implying that the trunk was pulled by the shoulder joint before the $11^{\text {th }}$ second. Then, the shoulder joint pushed the trunk until fully standing. The vertical force, the dashed line Fy3, is positive, implying upward pushing. The moment, represented by the solid line M3, changed from positive to negative and then returned to positive again, illustrating rotational movement between the arm and the trunk. Therefore, the subject's upper limbs pushed his trunk from the sitting to the standing position. The other figures explain the dynamic properties of the other joints as well.

\section{CONCLUSIONS}

A five-segment biomechanical human body model was established through kinematics. By giving known values from transducers which are angular displacement at the lower limbs and reactions and moments at handle, the kinematics and dynamics of this model were solved using Mathematica. Experimental data concerning lower limbs and handle were input to the model and the output revealed and explained every detail of the patient's sit-to-stand 


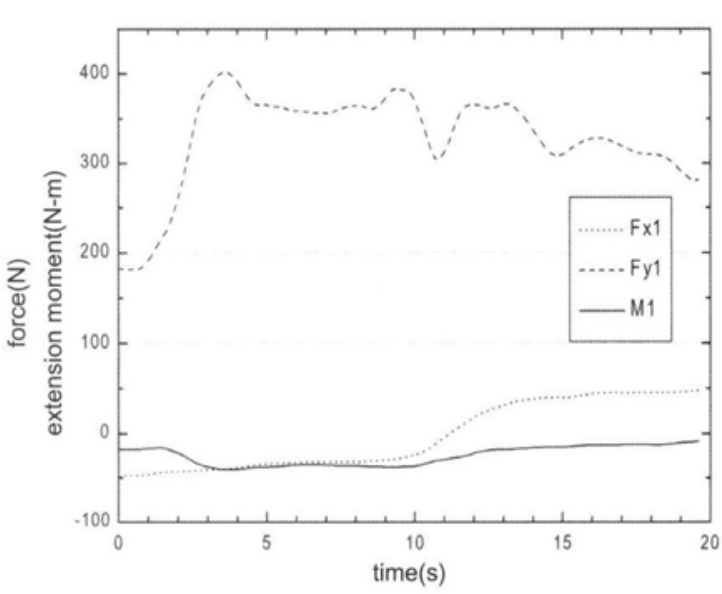

Fig. 8. Force and moment at wrist joint

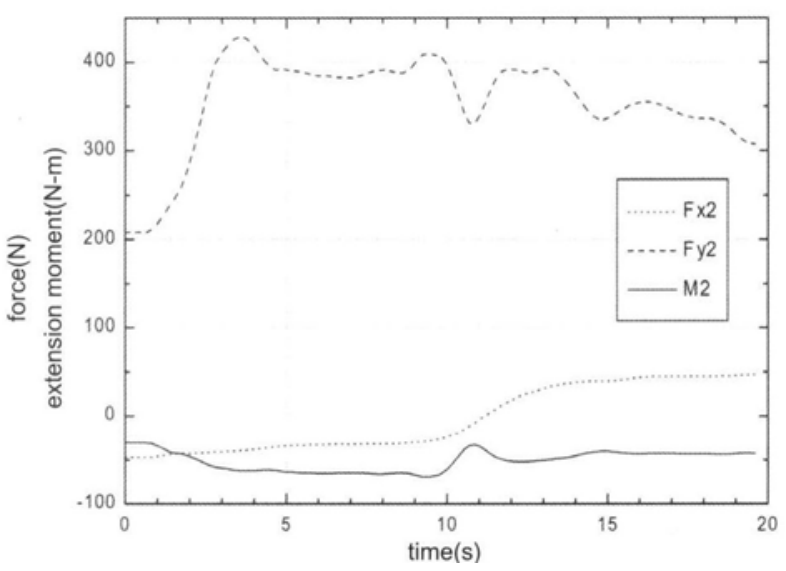

Fig. 9. Force and moment at elbow joint

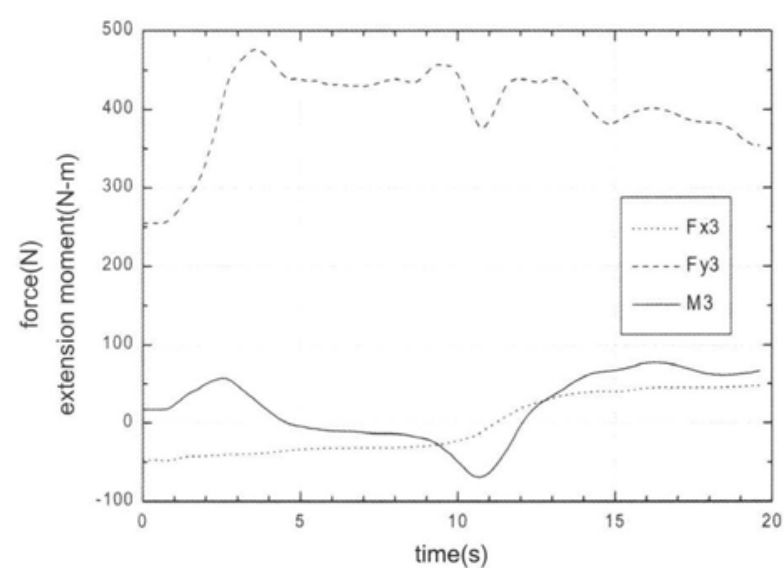

Fig. 10. Force and moment at shoulder joint

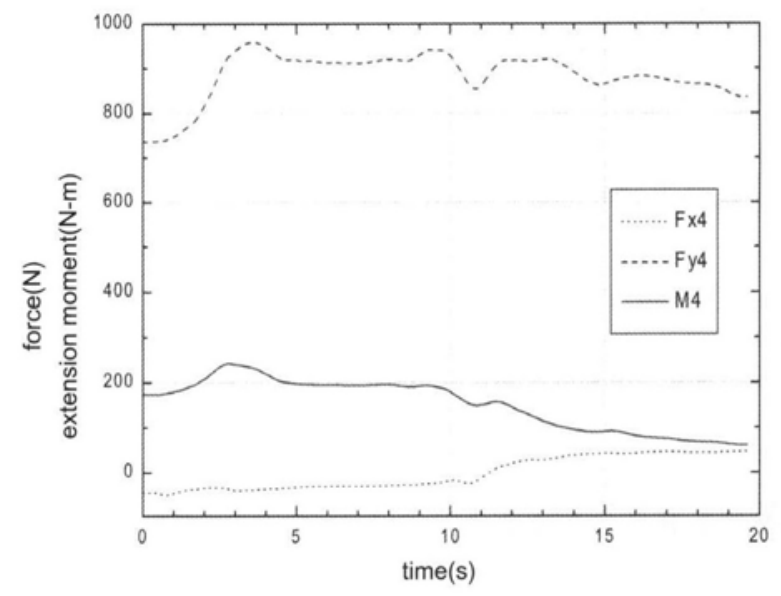

Fig. 11. Force and moment at hip joint

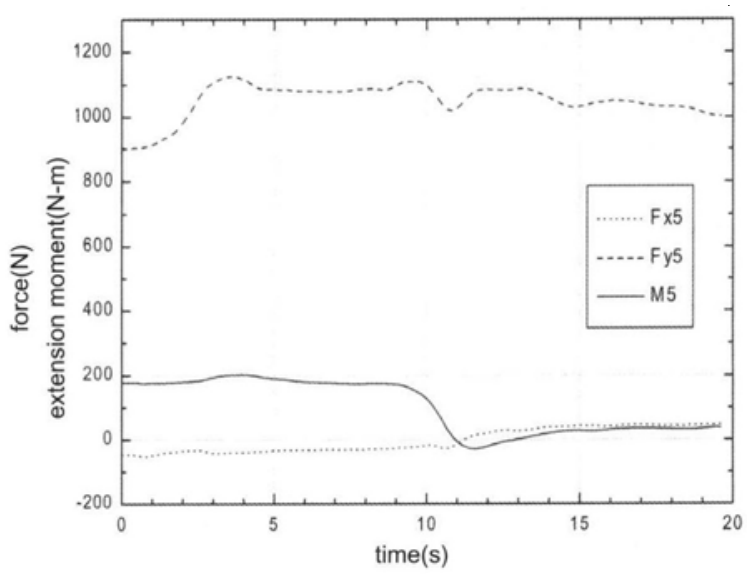

Fig. 12. Force and moment at knee joint

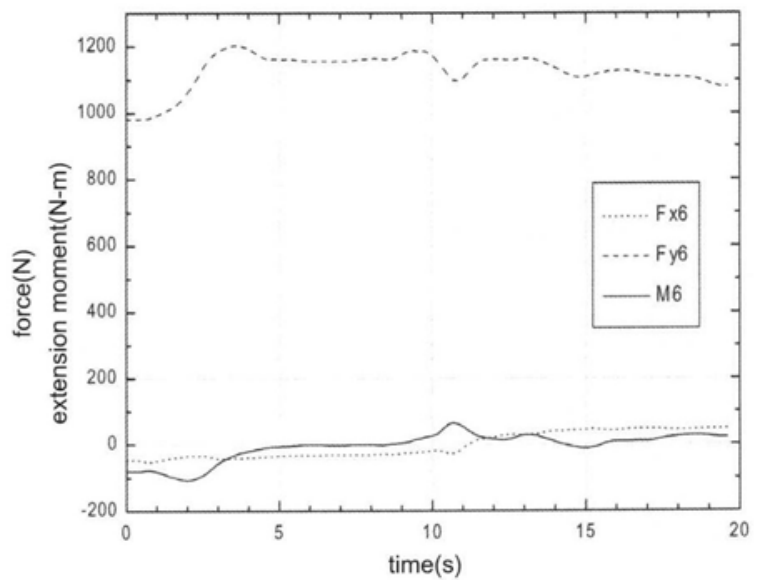

Fig. 13. Force and moment at ankle joint 
dynamics. This biomechanical model may help the understanding of the sit-to-stand process of SCI patients so as to benefit their rehabilitation therapy plan in the future.

\section{ACKNOWLEDGEMENTS}

The authors would like to thank the National Science Council of the Republic of China for financially supporting this research under the Contract No. NSC-90-2213-E-038-009.

\section{REFERENCEST}

1. Janssen, W. G. M., Bussmann, H. B. J., and Stam, H. J., "Determinants of the sit-to-stand movement: A review," Physical Therapy, Vol. 82, No. 9, pp. 866 879 (2002).

2. Roberts, P. D., and McCollum, G., "Dynamics of the sit-to-stand movement," Biological Cybernetics, Vol. 74, No. 2, pp. 147 157 (1996).

3. Johnson, M. W., and Peckham, P. H., "Evaluation of shoulder movement as a command control source," IEEE Trans. Biomed. Eng., Vol. 37, pp. 876 885 (1990).

4. Hughes, M. A., Myers, B. S., and Schenkman, M. L., "The role of strength in rising from a chair in the functionally impaired elderly," J. Biomechanics, Vol. 29, No. 12, pp. 1509 1513 (1996).

5. Kerr, K. M., White, J. A., Barr, D. A., and Mollan, R. A. B., "Analysis of the sit-stand-sit movement cycle in normal subjects," Clinical Biomechanics, Vol. 12, No. 4, pp. 236 245 (1997).

6. Munro, B. J., Steele, J. R., Bashford, G.M., Ryan, M., and Britten, N., "A kinematic and kinetic analysis of the sit-to-stand transfer using an ejector chair: implications for elderly rheumatoid arthritic patients," J. Biomechanics, Vol. 31, No. 3, pp. 263 271 (1998).

7. Bhadra, N., and Peckham, P. H., "Peripheral nerve stimulation for restoration of motor function," J. Clinical Neurophysiology, Vol. 14, No.5, pp. 378 393 (1997).

8. Kralj, A. and Bajd, T., Functional Electrical Stimulation: Standing and Walking After Spinal Cord Injury, Boca Raton, FL: CRC Press (1989).

9. Petrofsky, J. S., Heaton, H. H., and Phillips, C. A., "Outdoor bicycle for exercise in paraplegics and quadriplegics," J. Biomed. Eng., Vol. 5, pp. 292 296 (1983).
10. Ewins, D. J., Taylor, P. N., Crook, S. E., Lipczynski, R. T., and Swain, I. D., "Practical low cost stand/sit system for mid-thoracic paraplegics," J. Biomed. Eng., Vol. 10, pp. 184 188 (1988).

11. Andrews, B. J., Baxendale, R. H., Barnett, R., Phillips, G. F., Yamazaki, T., Paul, J. P., and Freeman, P.A., "Hybrid FES orthosis incorporating closed loop and sensory feedback," J. Biomed. Eng., Vol. 10, pp. 189 195 (1988).

12. Khang, G., and Zajac, F. E., "Paraplegic standing controlled by functional neuromuscular stimulation: part I - computer model and controlsystem design," IEEE Trans. Biomed. Eng., Vol. 36, pp. 873 884 (1989).

13. Graupe, D., Kohn, K. H., Kralj, A., and Basseas, S., "Patient controlled electrical stimulation via EMG signature discrimination for providing certain paraplegics with primitive walking functions," J. Biomed. Eng., Vol. 5, pp. 220 226 (1983).

14. Veltink, P. H., and Donaldson, N., "A perspective on the control of FES-supported standing," IEEE Trans. Rehabil. Eng., Vol. 6, No.2, pp. 109 112 (1998).

15. Donaldson, N., and Yu, C-h., "xperiments with CHRELMS patient-driven stimulator controllers for the restoration of function to paralysed legs,". Proceedings of Instrumentations Mechanical Engineers, Vol. 214, part H, pp.1 20 (2000).

16. Riener, R. and Fuhr, T., "Patient-driven control of FES-supported standing up: a simulation study," IEEE Trans. Rehab. Eng., Vol. 6, pp. 113 124 (1998).

17. Kralj, A., Jaeger, R. J., and Munih, M., "nalysis of standing up and sitting down in humans: definitions and normative data presentation," J. Biomech., Vol. 23, pp. 1123 1138 (1990).

18. Schenkman, M., Berger, R. A., Riley, P. O., Mann, R. W., and Hodge, W. A., "Whole-body movements during rising to standing from sitting," Physical Therapy, Vol. 70, pp. 638 648 (1990).

19. Doorenbosch, C. A. M., Harlaar, J., Roebroeck, M. E., and Lankhorst, G. J., "wo strategies of transferring from sit- to-stand; the activation of monoarticular and biarticular muscles," J. Biomech., Vol. 27, pp. 1299 1307 (1994).

20. Oyama, , Maeda, T., Tachi, S., "Human system learning model for solving the inverse kinematics problem by direct inverse modeling," Systems and Computers in Japan, Vol. 27, No. 8, pp. 53-68 (1996).

21. Yang, Z. Mi, J., Abdel-Malek, K., Mun, J., Nebel, K., "Real-time inverse kinematics for humans," Proceedings of the ASME Design Engineering Technical Conference, Vol. 5A, pp. 349-359 (2002). 
22. Tolani, D., Goswnmi, A., Badler, N., "Real-time inverse kinematics techniques for anthropomorphic limbs," Graphical Models, Vol. 62, No. 5, pp. 353$388(2000)$.

23. Lundin, T. M., Grabiner, M. D., and Jahnigen, D. W., "On the assumption of bilateral lowerextremity joint moment symmetry during the sit-tostand task," J. Biomech., Vol. 28, No. 1, pp. 109 112 (1995). 